\title{
A DIVERGENCE THEOREM FOR HILBERT SPACE
}

\author{
BY \\ VICTOR GOODMAN( $\left.{ }^{1}\right)$
}

\begin{abstract}
Let $B$ be a real separable Banach space. A suitable linear imbedding of a real separable Hilbert space into $B$ with dense range determines a probability measure on $B$ which is known as abstract Wiener measure. In this paper it is shown that certain submanifolds of $B$ carry a surface measure uniquely defined in terms of abstract Wiener measure. In addition, an identity is obtained which relates surface integrals to abstract Wiener integrals of functions associated with vector fields on regions in $B$. The identity is equivalent to the classical divergence theorem if the Hilbert space is finite dimensional. This identity is used to estimate the total measure of certain surfaces, and it is established that in any space $B$ there exist regions whose boundaries have finite surface measure.
\end{abstract}

1. Introduction. Let $B$ be a real separable Banach space with norm $\|\cdot\|$. Consider a linear imbedding $i$ of a real separable Hilbert space into $B$ with dense range. Gross [5] has shown that if the function $\|i \cdot\|$ is a measurable norm on the Hilbert space, then the space $B$ carries a Borel probability measure $p_{1}$ uniquely characterized by the identity

$$
p_{1}\left(\{x \in B:\langle y, x\rangle\langle r\})=\left(2 \pi\left|i^{*} y\right|^{2}\right)^{-1 / 2} \int_{-\infty}^{r} \exp \left[-\left(2\left|i^{*} y\right|^{2}\right)^{-1} s^{2}\right] d s .\right.
$$

Here, $y$ is any element of the topological dual space of $B, i^{*}$ is the adjoint of the imbedding map, and $|\cdot|$ is the norm on the dual Hilbert space. The space $B$, together with the Hilbert space and the imbedding, is said to be an abstract Wiener space, and $p_{1}$ is said to be abstract Wiener measure on $B$.

The above measure is easily identified if the associated Hilbert space is finite dimensional. Since any dense linear imbedding of a finite dimensional space is invertible, a range space $B$ of such an imbedding is isomorphic to the Hilbert space. Under the isomorphism, the measure $p_{1}$ is given by

$$
p_{1}=(2 \pi)^{-\mathrm{dim} B / 2} \exp \left[-|x|^{2} / 2\right] d x
$$

where $d x$ is Lebesgue measure on the Hilbert space and $|\cdot|$ is the Hilbert norm.

Received by the editors January 4, 1971.

AMS 1970 subject classifications. Primary 26A96, 26A66, 28A40; Secondary 26A60, 28A25, 46G99.

Key words and phrases. Integral calculus on Banach spaces, abstract Wiener spaces, Hilbert space, divergence theorem, Gauss' theorem.

(1) Some of the results in this paper appeared in the author's doctoral dissertation at Cornell University, prepared under the supervision of Professor L. Gross; the research was partially supported by NSF Grant GU-2582.

Copyright (C) 1972, American Mathematical Society 
Certain features of the integral calculus for the finite dimensional example above have been abstracted to infinite dimensional spaces. However, the imbeddings which arise in the infinite dimensional case are not invertible and the topology of the range space of an imbedding enters nontrivially into the formulation of such abstractions. For example, it is known that a closed subspace with finite codimension in an abstract Wiener space is itself an abstract Wiener space and Wiener measure is a product of a measure on the subspace and a measure on a supplementary subspace. We use this fact to prove that certain (nonlinear) submanifolds of any abstract Wiener space carry a uniquely defined surface measure.

An important regularity condition which is defined for a map $F$ from an abstract Wiener space $B$ to a Banach space $Y$ is differentiability in Hilbert space directions. Let $i$ denote the imbedding of the associated Hilbert space $H$. For fixed $x$ in $B$, the auxiliary function $V(h)=F(x+i h)$ is defined on $H$. If $V: H \rightarrow Y$ is Fréchet differentiable at the origin, then $F$ is said to be differentiable in Hilbert space directions at the point $x$. We shall say that the function $F$ is $H$ differentiable at $x$. We define the divergence of certain $H$ differentiable vector fields on a space $B$, and we derive an identity which relates the Wiener integral of the divergence over a region to a surface integral over the boundary of the region. Our use of the Hilbert space imbedding makes clear the fact that the integrals are determined by the Hilbert inner product and the derivative of the vector field in Hilbert space directions. Hence, we refer to the integral identity as a divergence theorem for Hilbert space.

It is well known that certain Banach spaces do not admit Fréchet differentiable partitions of unity. However, in an abstract Wiener space the condition that a function be $H$ differentiable is less stringent than the condition for Fréchet differentiability, and we show that nontrivial $H$ differentiable partitions of unity exist. Such partitions are used in establishing the divergence theorem. In connection with this technical problem we remark that the surfaces considered in the paper are of a more general type than Fréchet differentiable surfaces. It is shown that these surfaces exist even in Banach spaces that do not admit Fréchet differentiable partitions of unity.

If a real-valued function $u$ on a real Hilbert space is twice Fréchet differentiable at a point $x$, then the second derivative $u^{\prime \prime}(x)$ may be identified with a bounded operator on the space. Gross [6] has defined the Laplacian of $u$ by

$$
\Delta u(x)=\operatorname{trace}\left[u^{\prime \prime}(x)\right]
$$

if $u$ has the property that $u^{\prime \prime}(x)$ is a trace class operator (or equivalently, a nuclear operator [3]). A similar definition has been formulated for certain $H$ differentiable functions on an abstract Wiener space. For such a function, the vector field $u^{\prime}$ is defined in a neighborhood of $x$ and our definition of the divergence of $u^{\prime}$ coincides with the definition of the Laplacian of $u$. We apply the divergence theorem to vector fields of the form $u^{\prime}$ to relate the Wiener integral of the Laplacian of $u$ to a boundary integral. This relation is used to establish the existence of surfaces in abstract Wiener spaces which have finite surface measure. 
2. Preliminaries. In dealing with an abstract Wiener space it is convenient to identify the associated Hilbert space with its image in the Banach space. In the paper, the pair $(H, B)$ will always denote an abstract Wiener space where $H$ is a dense subspace of $B$ with a Hilbert norm $|x|=(x, x)^{1 / 2}$.

Definition 1. Let $(H, B)$ be an abstract Wiener space. A map $F: E \rightarrow Y$ from a subset $E$ of $B$ into a Banach space $Y$ is said to be $H$ continuous at $x$ if the map $V(h)=F(x+h)$, defined on $(E-x) \cap H$, is continuous at the origin in the relativized Hilbert topology. A map $F$ on an open set $E$ in $B$ is $H$ differentiable at $x$ if the function $V$ is Fréchet differentiable at the origin in $H$. If $V^{\prime}(0)$ exists, then this operator is said to be the $H$ derivative of $F$ at $x$.

If a real-valued function $u$ defined on an open subset of $B$ is $H$ differentiable at $x$, we denote by $(D u)(x)$ the $H$ derivative at $x$. Higher $H$ derivatives of $u$ may be defined inductively using definition one. We denote by $D^{n} u(x)$ the $n$th $H$ derivative of $u$ at $x$ if it exists.

Suppose that $p_{1}$ is abstract Wiener measure on the space $(H, B)$. For fixed $x$ in $B$ and a Borel subset $E$ of $B$, the equation

$$
p_{t}(x, E)=p_{1}\left(t^{-1 / 2} E-x\right), \quad t>0,
$$

defines a Borel probability measure $p_{t}(x$,$) on B$. An alternate definition of this measure is given in [6, p. 127]. It follows from equation (3) in [6] that the two definitions are equivalent. The measure $p_{t}(0$,$) is denoted by p_{t}()$, and this measure is said to be abstract Wiener measure with variance parameter $t$. If $f$ is a bounded measurable real-valued function on $B$ the function $p_{t} f$ is defined by

$$
\left(p_{t} f\right)(x)=\int_{B} f(y) p_{t}(x, d y)=\int_{B} f(x+y) p_{t}(d y) .
$$

By Proposition 9 of [6], such a function is infinitely $H$ differentiable on $B$.

For any space $(H, B)$, the imbedding $H \rightarrow B$ has dense range. Hence, the adjoint map $B^{*} \rightarrow H^{*}$ is injective. The use of this injection clarifies the manner in which the linear structure of $B$ is used in the integral calculus for abstract Wiener measure. First, the canonical isomorphism between $H^{*}$ and $H$ allows one to identify $B^{*}$ with a subspace of $H$ and, hence, of $B$. Thus, we have the inclusions $B^{*} \subset H \subset B$. Secondly, an element of the space $L\left(B, B^{*}\right)$, that is, a continuous linear operator from $B$ to $B^{*}$, may be identified with an element of $L(H, H)$. In view of the identifications above, this is accomplished by restricting the operator to $H$. The elements of $L\left(B, B^{*}\right)$ which we shall be interested in are those whose restrictions to $H$ are orthogonal projections on $H$.

One may easily see that elements of $B^{*} \cap H$ are characterized in terms of the norm $\|\cdot\|$ on $B$ as follows: An element $x$ of $H$ is in $B^{*}$ if and only if there exists a constant $C>0$ such that the inequality $|(x, y)| \leqq C\|y\|$ holds for all $y$ in $H$. For such an element $x$ we shall denote by $\langle x, \cdot\rangle$ the continuous extension of the linear functional $(x, \cdot)$ to all of $B$. 
RemarK 1. Let $K \subset H$ be a finite dimensional subspace of $B^{*}$ where $(H, B)$ is an abstract Wiener space. If $y_{1}, \ldots, y_{n}$ is an orthonormal basis of $K$ then the equation $Q x=\sum\left\langle y_{j}, x\right\rangle y_{j}$ defines a continuous operator from $B$ to $B^{*}$. Since $Q$ is the continuous extension of the orthogonal projection of $H$ onto $K, Q$ is a projection on $B$. Thus, $B=K \oplus L$ where $L$ is the null space of $Q$. Let $K^{\perp}$ denote the orthogonal complement of $K$ in $H$. By Remark 2.2 of [6], the pair $\left(K^{\perp}, L\right)$ is an abstract Wiener space and we have the product decomposition $p_{t}=p_{t}^{\prime} \times \mu_{t}$ where $p_{t}^{\prime}$ is abstract Wiener measure on $L$ and $\mu_{t}$ is Gauss measure on $K$. That is,

$$
\mu_{t}=(2 \pi t)^{-\mathrm{dim} K / 2} \exp \left[-|k|^{2} / 2 t\right] d k
$$

where $K$ is viewed as an inner product space.

\section{Surfaces in abstract Wiener spaces.}

Definition 2. Let $(H, B)$ be an abstract Wiener space. A real-valued function $g$ defined on an open subset $U$ of $B$ is an $H-C^{1}$ function if $g$ is continuous and $H$ differentiable on $U$, the map $D g: U \rightarrow H^{*}$ is continuous, and for each $x$ in $U$, the vector $D g(x)$ is in $B^{*}$.

Definition 3. Let $(H, B)$ be an abstract Wiener space. A subset $S$ of $B$ is an $H-C^{1}$ surface if for each $x$ in $S$ there is an open neighborhood $U$ of $x$ in $B$ and an $H-C^{1}$ function $g$ defined on $U$ such that $D g(x) \neq 0$ and $S \cap U=\{y \in U: g(y)=0\}$.

A subset $V$ of $B$ has an $H-C^{1}$ boundary if for each point $x$ in $\partial V$ there is a neighborhood $U$ of $x$ and a function $g$ with the above properties such that $V \cap U$ $=\{y \in U: g(y)<0\}$.

REMARK 2. Definition 2 differs from the standard definition of a differentiable surface in a Banach space in that the function $g$ appearing in Definition 2 is not required to be continuously Fréchet differentiable on its domain. One may easily verify that any differentiable surface in a space $B$ is an $H-C^{1}$ surface for the pair $(H, B)$. It is not known whether in every Banach space there exists a bounded set with a differentiable boundary. In fact, the works [1], [7] and [10] show that for many Banach spaces the set of continuously Fréchet differentiable functions is rather small. This suggests that for such spaces there do not exist many differentiable surfaces. Nevertheless, using abstract Wiener measure we show that for any pair $(H, B)$, the space $B$ has nonempty bounded open sets with $H-C^{1}$ boundaries.

Proposition 1. For an abstract Wiener space $(H, B)$ let $B_{r}(x)$ denote the ball of radius $r$ centered at $x$ in $B$. Then for each pair of numbers $0<r<r^{\prime}$, there exists a set $V$ with an $H-C^{1}$ boundary such that $B_{r}(x) \subset V \subset B_{r^{\prime}}(x)$.

Proof. Given numbers $r$ and $r^{\prime}$, choose $\varepsilon>0$ such that $\varepsilon<r<r^{\prime}<\varepsilon^{-1}$, and put $f(y)=\min \left\{\|y\|, 2 \varepsilon^{-1}\right\}$ where $\|y\|$ is the norm on $B$. By a remark above, the function $g_{t}=\left(p_{t} f\right)$ is $H$ differentiable on $B$ for each $t>0$. Since the function $f$ satisfies a Lipschitz condition on $B$ it follows that $g_{t}$ satisfies a Lipschitz condition for each $t$. That is, for some $M>0$ the inequality

$$
\left|g_{t}(y)-g_{t}(z)\right| \leqq M\|y-z\|
$$


holds for all $y$ and $z$ in $B$. But then for any element $h$ in $H$,

$$
\left|\left(D g_{t}(y), h\right)\right| \leqq \limsup _{s \rightarrow 0} s^{-1}\left|g_{t}(y+s h)-g_{t}(y)\right| \leqq M\|h\| .
$$

Hence, $D g_{t}(y)$ is in $B^{*}$ for each $y$ in $B$ and the $B^{*}$ norm is not greater than $M$. Also, from Proposition 9 of [6] we have

$$
\begin{aligned}
\left|\left(D g_{t}(y), h\right)-\left(D g_{t}(z), h\right)\right| & =\left|t^{-1} \int_{B}\{f(y+v)-f(z+v)\}(h, v) p_{t}(d v)\right| \\
& \leqq 2 t^{-1 / 2} M\|y-z\||h| .
\end{aligned}
$$

It follows that the map $D g_{t}: B \rightarrow H^{*}$ is continuous. By Proposition 6 of [6], the function $g_{t}$ converges uniformly to $f$ as $t$ approaches zero. We put $r^{\prime \prime}=\left(r+r^{\prime}\right) / 2$. Then for $t$ sufficiently small, the set $V_{t}=\left\{y: g_{t}(y)<r^{\prime \prime}\right\}$ satisfies $B_{r}(0) \subset V_{t} \subset B_{r^{\prime}}(0)$. To prove that $V_{t}$ is a set with an $H-C^{1}$ boundary for $t$ small, it suffices to show that $D g_{t}$ is nonzero on the set $\left\{y: \varepsilon<\|y\|<\varepsilon^{-1}\right\}$ for $t$ sufficiently small.

Given $y$ such that $\varepsilon<\|y\|<\varepsilon^{-1}$, choose $h$ in $H$ such that $\|h-y\|<\varepsilon / 4$. Then if $s$ is a positive number,

$$
s^{-1}\left\{g_{t}(y+s h)-g_{t}(y)\right\}=s^{-1} \int_{\|z\|<\varepsilon / 4}\{\|y+s h+z\|-\|y+z\|\} p_{t}(d z)+E(s)
$$

where $E(s)=s^{-1} \int_{\|z\| \geqq \varepsilon / 4}\{f(y+s h+z)-f(y+z)\} p_{t}(d z)$. Since $f$ satisfies a Lipschitz condition we have $|E(s)| \leqq M\|h\| p_{t}(\{\|z\| \geqq \varepsilon / 4\})$. One may verify directly that $\|y+s h+z\|-\|y+z\| \geqq \varepsilon s / 4$ if $\|z\|<\varepsilon / 4$. Hence by letting $s$ approach zero we obtain

$$
\left(D g_{t}(y), h\right) \geqq(\varepsilon / 4) p_{t}\left(\{\|z\|<\varepsilon / 4\}-M\|h\| p_{t}(\{\|z\| \geqq \varepsilon / 4\}) .\right.
$$

But $\lim _{t \downarrow o} p_{t}(\{\|z\|<\varepsilon / 4\})=1$ and thus $D g_{t}(y)$ is nonzero for $t$ sufficiently small. Moreover, a uniform estimate as above may be made on the set $\left\{y: \varepsilon<\|y\|<\varepsilon^{-1}\right\}$ since the only requirement for $h$ is that $\|y-h\|<\varepsilon / 4$. Hence $V_{t}$ has an $H-C^{1}$ boundary and clearly any translate of this set also has an $\mathrm{H}-\mathrm{C}^{1}$ boundary.

Corollary OF THE PRoOF. Let $\|\cdot\|$ denote the $B$ norm for a space $(H, B)$. For any $\varepsilon>0$ there exists a bounded function $f: B \rightarrow R$ satisfying a Lipschitz condition on $B$ such that the functions $p_{t}$ f converge uniformly to the norm on the set $\left\{y:\|y\|<\varepsilon^{-1}\right\}$ as $t \downarrow 0$, and $D p_{t}$ fis nonzero on the set $\left\{x: \varepsilon<\|x\|<\varepsilon^{-1}\right\}$ for $t$ sufficiently small.

Proof. By the proof of the proposition the statement holds for

$$
f(x)=\min \left\{\|x\|, 2 \varepsilon^{-1}\right\} .
$$

This corollary will be used in a later argument.

Definition 4. Let $K$ be a differentiable surface in a real Hilbert space $H$. A one dimensional orthogonal projection $P$ on $H$ is a normal projection for $K$ at $x$ in $K$ if there holds

for all $y$ in $K$.

$$
|P(y-x)|=o(|y-x|), \quad|y-x| \downarrow 0,
$$


REMARK 3. If $K$ is a differentiable surface in a Hilbert space, the condition that a one dimensional projection $P$ is a normal projection at $x$ is equivalent to the condition that the hyperplane $x+\operatorname{ker} P$ is tangent to $K$ at $x$. It follows from the implicit function theorem [2, Theorem 10.2.1], that such a projection exists for each element of a differentiable surface and that each projection is unique.

REMARK 4. If $S$ is an $H$ - $C^{1}$ surface in the space $(H, B)$, it is an immediate consequence of Definition 3 that for any $x$ in $S$ the set $(S-x) \cap H$ is a differentiable surface in $H$.

Proposition 2. Let $S$ be an $H-C^{1}$ surface in an abstract Wiener space $(H, B)$. There exists a unique map $N: S \rightarrow L\left(B, B^{*}\right)$ such that

(i) for each $x$ in $S$, the restriction of $N_{x}$ to $H$ is a normal projection for $(S-x) \cap H$ at 0 ,

(ii) for each $x$ the map $J_{x}=I-N_{x}$ is a homeomorphism of an open neighborhood in $S$ of $x$ onto an open subset in the null space of $N_{x}$, and

(iii) the map $N: S \rightarrow L(H, H)$ (with the operator norm topology on the range) is continuous.

Proof. Suppose $x$ is in $S$. Since $(S-x) \cap H$ is a differentiable surface in $H$ containing 0 , by Remark 2 there exists a unique normal projection at 0 . Thus, if the map $N$ exists, it is uniquely determined by property (i).

For $x$ in $S$ let $U$ and $g$ be as in Definition 3. Since $D g: U \rightarrow H^{*}$ is continuous we may assume with no loss of generality that $|D g|>0$ on $U$. We put $v(h)=g(h+x)$ for $h$ in $(U-x) \cap H$. Since $v$ is continuously Fréchet differentiable on this set and $(S-x) \cap H$ is a level surface of $v$ on this set, we obtain

$$
N_{x}=|D v(0)|^{-2}(D v(0), \cdot) D v(0)=|D g(x)|^{-2}(D g(x), \cdot) D g(x)
$$

by an elementary argument. By assumption, $D g(x)$ is in $B^{*}$ and so $N_{x}$ extends to a continuous projection on $B$. Moreover, for any $y$ in $U$ the same argument yields that the equality holds for $x$ replaced by $y$. Hence, property (iii) follows from the continuity of the map $D g: U \rightarrow H^{*}$.

We put $h=|D g(x)|^{-1} D g(x)$ so that $N_{x}=\langle h, \cdot\rangle h$. If $L_{x}$ denotes the null space of $N_{x}$ we have $L_{x}=\{y \in B:\langle h, y\rangle=0\}$. Then $B=L_{x} \oplus \operatorname{span}\{h\}$. Now $x=l_{0}+s_{o} h$ for some $l_{o}$ in $L_{x}$ and $s_{o}$ in $R$. We define a function $w$ in a neighborhood in $L_{x} \times R$ of $\left(l_{o}, s_{o}\right)$ by setting $w(l, s)=s-|D g(x)|^{-1} g(l+s h)$. Using the fundamental theorem of calculus one sees that $w$ is a contraction mapping in the second variable in a neighborhood of $\left(l_{o}, s_{o}\right)$. We apply [2, Theorem 10,1.1] to obtain a continuous realvalued function $u$ defined in an $L_{x}$ neighborhood of $l_{o}$ satisfying $g(l+u(l) h)=0$. Hence, the map $l \rightarrow l+u(l) h$ is continuous into $S \cap U$. This map has a continuous inverse, for $J_{x}(l+u(l) h)=l$. Also, by the uniqueness part of Theorem 10.1.1 the map $J_{x}$ followed by $l+u(l) h$ is the identity map on some open neighborhood in $S$ of $x$. This establishes that $J_{x}$ is a local homeomorphism into $L_{x}$. 
Definition 5. Let $S$ be an $H-C^{1}$ surface in an abstract Wiener space $(H, B)$. The map $N$ whose existence and uniqueness is asserted in Proposition 2 is the normal projection for $S$. If $y$ is in $S$ then by Proposition 2 there is an element $h$ in $B^{*}$ of unit Hilbert norm and a neighborhood $W$ in $S$ of $y$ such that

(i) $N_{y} h=h$,

(ii) $\left|N_{w} h\right|>0$ for all $w$ in $W$, and

(iii) $J_{y}=I-N_{y}$ is a homeomorphism of $W$ into the null space of $N_{y}$.

The element $h$ is said to be a unit normal at $y$ and $W$ is said to be a coordinate neighborhood of $y$.

\section{Surface measure.}

Definition 6. Let $S$ be an $H-C^{1}$ surface in an abstract Wiener space $(H, B)$, and let $W$ be a coordinate neighborhood of a point $y$ in $S$. For a fixed $x$ in $B$ and $t>0$ we define a measure $\rho(t, x, W, \cdot)$ on the Borel sets of $W$ by

$$
\rho(t, x, W, E)=(2 \pi t)^{-1 / 2} \int_{J_{y}(E)}\left|N_{w} h\right|^{-1} \exp \left[-(2 t)^{-1}\left|N_{y}(w-x)\right|^{2}\right] p_{t}^{\prime}\left(J_{y} x, d l\right) .
$$

In this formula $h$ is a unit normal at $y, p_{t}^{\prime}(\cdot)$ is Wiener measure on the null space of $N_{y}$, and $w=J_{y}^{-1} l . \rho(t, x, W, \cdot)$ is said to be a local version of normal surface measure with dilation parameter $t$ and translation variable $x$.

REMARK 5. It is instructive to identify the measures given by Definition 6 when $H$ is the finite dimensional inner product space $R^{n}$. For such a space the Euclidean norm is measurable and an $H-C^{1}$ surface $S$ is a differentiable surface in $R^{n}$. It follows immediately from the formula in Definition 6 that

$$
\rho(t, x, W, \cdot)=(2 \pi t)^{-n / 2} \exp \left[-(2 t)^{-1}|s-x|^{2}\right] d s
$$

where $d s$ is (Lebesgue) surface measure on $S$.

LEMMA 1.1. Let $S$ be an $H-C^{1}$ surface for the space $(H, B)$ and let $y$ and $z$ be two points in $S$. If for fixed $x$ in $B, \rho(t, x, W, \cdot)$ and $\rho\left(t, x, W^{\prime}, \cdot\right)$ are local versions of normal surface measure on coordinate neighborhoods $W, W^{\prime}$ of $y, z$ respectively, then for any Borel set $E \subset W \cap W^{\prime}$ there holds $\rho(t, x, W, E)=\rho\left(t, x, W^{\prime}, E\right)$.

Proof. Suppose $W \cap W^{\prime} \neq \varnothing$. Let $h_{y}, h_{z}$ be unit normals at $y, z$ and let ${ }_{y} p_{t}^{\prime},{ }_{z} p_{t}^{\prime}$ denote Wiener measure on the null spaces $L$ and $L^{\prime}$ of $N_{y}$ and $N_{z}$. By Definition 6,

$$
\rho\left(t, x, W^{\prime}, E\right)=(2 \pi t)^{-1 / 2} \int_{J_{z}(E)}\left|N_{w^{\prime}} h_{z}\right|^{-1} \exp \left[-(2 t)^{-1}\left|N_{z}\left(w^{\prime}-z\right)\right|^{2}\right]_{z} p_{t}^{\prime}\left(J_{z} x, d l^{\prime}\right)
$$

where $w^{\prime}=J_{z}^{-1} l^{\prime}$. Since $T=J_{z} J_{y}^{-1}$ is a homeomorphism on $J_{y}\left(W \cap W^{\prime}\right)$, the integral may be written as

$$
(2 \pi t)^{-1 / 2} \int_{J_{y}(E)}\left|N_{w} h_{z}\right|^{-1} \exp \left[-(2 t)^{-1}\left|N_{z}\left(J_{y}^{-1} l-x\right)\right|^{2}\right]_{z} p_{t}^{\prime}\left(J_{z} x, T(d l)\right),
$$

where $w=J_{y}^{-1} l$. 
We consider the measure ${ }_{z} p_{t}^{\prime}\left(J_{z} x, T(d l)\right)$ on $J_{y}\left(W \cap W^{\prime}\right)$. If $h_{y}$ and $h_{z}$ are linearly dependent then $N_{y}=N_{z}$ and the equality follows immediately. Hence, we may assume that $J_{y} h_{z}=h_{z}-N_{y} h_{z} \neq 0$. Since $J_{y} h_{z}$ is in $B^{*}$ and $J_{y} h_{z}$ vanishes on $M=L_{y} \cap L_{z}$ we have $L_{y}=M \oplus \operatorname{span}\{v\}$ where $v=\left|J_{y} h_{z}\right|^{-1} J_{y} h_{z}$. Similarly, $L_{z}$ $=M \oplus \operatorname{span}\{k\}$ where $k=\left|J_{z} h_{y}\right|^{-1} J_{z} h_{y}$. We derive an expression for $T(l)$ using the direct sum decomposition $l=m+s v, m \in M, s \in R$. Using the identities $J_{y}\left(J_{y}^{-1} l\right)$ $=l$ and $N_{y}=\left(h_{y}, \cdot\right) h_{y}$ we obtain $J_{y}^{-1} l=l+\left(h_{y}, J_{y}^{-1} l\right) h_{y}$ and thus $T(l)=J_{z} J_{y}^{-1}(l)$ $=J_{z} l+\left(h_{y}, J_{y}^{-1} l\right) J_{z} h_{y}=m+\left\{\left|J_{z} h_{y}\right|\left(h_{y}, J_{y}^{-1} l\right)-\left(h_{y}, h_{z}\right)\right\} k$.

We put $\alpha(m, s)=\left|J_{z} h_{y}\right|\left(h_{y}, J_{y}^{-1} l\right)-\left(h_{y}, h_{z}\right)$. By Remark $1,{ }_{z} p_{t}^{\prime}=p_{t}^{\prime \prime} \times \mu_{t}$ where $p_{t}^{\prime \prime}$ is Wiener measure on $M$ and $\mu_{t}$ is Gauss measure on span $\{k\}$. By applying the one dimensional Jacobi theorem one obtains

$$
{ }_{z} p_{t}^{\prime}(T(d l))=(2 \pi t)^{-1 / 2} \exp \left[-(2 t)^{-1} \alpha(m, s)^{2}\right](\partial \alpha / \partial s)(m, s) p_{t}^{\prime \prime}(d m) d s
$$

where $d s$ is Lebesgue measure on $\operatorname{span}\{k\}$.

Given any $l_{o}$ in $J_{y}\left(W \cap W^{\prime}\right)$, by Definition 3 and Proposition 2 there is an $H$ differentiable function $g$ on a neighborhood of $J_{y}^{-1} l_{o}$ such that $g\left(l+u(l) h_{y}\right)=0$ where $u(l)=\left(h_{y}, J_{y}^{-1} l\right)$ for $l$ in a neighborhood of $l_{0}$. By the implicit function theorem, $\partial u / \partial s=-\left|N_{x} h_{y}\right|^{-1}\left|N_{w} v\right|, w=J_{y}^{-1} l$, and it follows that

$$
(\partial \alpha / \partial s)(l)=\left|N_{w} h_{y}\right|^{-1}\left|N_{w} h_{z}\right|
$$

We replace ${ }_{z} p_{t}^{\prime}\left(J_{z} x, T \cdot\right)$ in (1) by the formula for this measure derived above. The resulting expression is the formula for $\rho(t, x, W, E)$.

THEOREM 1. Let $S$ be an $H-C^{1}$ surface in an abstract Wiener space $(H, B)$. For $x$ in $B$ and $t>0$ there is a unique positive measure $\sigma_{t}(x, \cdot)$ on the Borel field of $S$ such that for any local version of normal surface measure, $\rho(t, x, W, \cdot)$, on a coordinate neighborhood $W$ in $S$ and any Borel subset $E$ of $W$ there holds $\sigma_{t}(x, E)=\rho(t, x, W, E)$.

Proof. If $S$ is an $H-C^{1}$ surface then the collection of all open coordinate neighborhoods of points of $S$ is an open cover of $S$. Since $S$ is a subset of a separable metric space there is a countable subcover. We choose such a cover, $\left\{W_{n}\right\}$, and construct a disjoint cover $\left\{Y_{n}\right\}$ of Borel sets with the property that $Y_{n} \subset W_{n}$. We define a set function $\sigma_{t}(x, \cdot)$ on the Borel field of $S$ by setting

$$
\sigma_{t}(x, E)=\sum_{1}^{\infty} \rho\left(t, x, W_{n}, E \cap Y_{n}\right)
$$

where $\rho\left(t, x, W_{n}, \cdot\right)$ is given by Definition 6 . It follows from the countable additivity of each local version that $\sigma_{t}(x, \cdot)$ is countably additive.

Now suppose that $\rho(t, x, W, \cdot)$ is a local version of normal surface measure on an arbitrary coordinate neighborhood $W$. If $E \subset W$ is a Borel set, we have

$$
\sigma_{t}(x, E)=\sum_{1}^{\infty} \rho\left(t, x, W_{n}, E \cap Y_{n}\right)
$$


But $E \cap Y_{n}$ is a subset of $W_{n} \cap W$ and, by Lemma 1.1, $\rho\left(t, x, W_{n}, E \cap Y_{n}\right)$ $=\rho\left(t, x, W, E \cap Y_{n}\right)$. Hence, $\sigma_{t}(x, E)=\rho(t, x, W, E)$. The uniqueness of the measure $\sigma_{t}(x, \cdot)$ follows from the equality above.

Definition 7. Let $S$ be an $H-C^{1}$ surface in an abstract Wiener space $(H, B)$. For $x$ in $B$ and $t>0$, the measure whose existence and uniqueness is shown in Theorem 1 is the normal surface measure on $S$ with dilation parameter $t$ and translation variable $x$.

DEFINITION 8. Let $V$ be an open set with $H-C^{1}$ boundary in an abstract Wiener space $(H, B)$. A map $n: \partial V \rightarrow H^{*}$ with the property that $n(y)$ is a unit normal for the surface $\partial V$ at $y$ and $y-\operatorname{sn}(y)$ is in $V$ for small $s>0$ is said to be an outward normal map for $V$.

It follows directly from Definition 3 and the proof of Proposition 2 that an outward normal map exists for a set with $H-C^{1}$ boundary and that the map is unique. Moreover, by the proof of Proposition 2 the map is continuous on the boundary of the set into $H^{*}$.

\section{The divergence theorem.}

Definition 9. Let $(H, B)$ be an abstract Wiener space. A sequence $\left\{\gamma_{n}\right\}$ of realvalued functions on $B$ is said to be an $H-C^{1}$ partition of unity if for each $n, \gamma_{n}: B \rightarrow[0,1]$ is an $H-C^{1}$ function, for each $x$ in $B$ there is a neighborhood $U$ of $x$ on which only finitely many $\gamma_{n}$ are nonzero, and $\sum_{1}^{\infty} \gamma_{n}(x)=1$.

LEMMA 2.1. Let $(H, B)$ be an abstract Wiener space. If $\left\{U_{\alpha}\right\}_{\alpha \in \mathscr{A}}$ is any open cover of $B$ there exists an $H-C^{1}$ partition of unity, $\left\{\gamma_{n}\right\}$, such that for each $n$, the support of $\gamma_{n}$ is contained in some element of the cover.

Proof. Let $f: B \rightarrow R$ be a bounded function which satisfies a Lipschitz condition on $B$. For $t>0$ the function $p_{t} f$ is an $H-C^{1}$ function by an argument in the proof of Proposition 1. Also, by Proposition 6 of [6], the family $\left\{p_{t} f\right\}$ converges to $f$ uniformly as $t \downarrow 0$. Hence, any bounded Lipschitz function on $B$ is the uniform limit of $H-C^{1}$ functions. Let $B_{r}(x)$ denote the ball of radius $r$ centered at $x$ in $B$. By the argument above, for any $r^{\prime}<r$ there is an $H-C^{1}$ function which vanishes outside the set $B_{r}(x)$ and is equal to one on the set $B_{r^{\prime}}(x)$. It follows from a standard argument that for any open cover of $B$ there exists a subordinate $H-C^{1}$ partition of unity. An example of such an argument appears in [8, pp. 29-30].

LEMMA 2.2. Let $(H, B)$ be an abstract Wiener space. For any $x$ in $B$ there exists a sequence of twice $H$ differentiable measurable functions $\left\{g_{n}\right\}, g_{n}: B \rightarrow[0,1]$ such that for each $n$ the support of $g_{n}$ is compact in $B,\left|D g_{n}\right| \leqq 1$ on $B,\left\{\left|D g_{n}\right|\right\}$ converges to zero almost everywhere with respect to $p_{t}(x, \cdot)$ and $\left\{g_{n}\right\}$ converges to 1 almost everywhere with respect to $p_{t}(x, \cdot)$ and $\sigma_{t}(x, \cdot)$, where $\sigma_{t}(x, \cdot)$ is the normal surface measure on any $H-C^{1}$ surface in $B$.

Proof. Let $\|\cdot\|$ denote the $B$ norm. It follows from the reflexivity of $H$ that the image of $B^{*}$ is dense in $H^{*}$. Hence, there is a sequence of finite dimensional 
projections on $H$ which converges strongly to the identity and has the property that each projection extends to a continuous projection on $B$. By Corollary 5.2 of [4] and the proofs of Lemma 1 and Lemma 2 of [5], there exists a sequence $\left\{\|\cdot\|_{j}\right\}$ of continuous tame seminorms on $B$ such that $\sum_{1}^{\infty}\|\cdot\|_{j}$ is a measurable norm on $H$ and $K_{r}=\left\{y \in B: \sum_{1}^{\infty}\|y\|_{j}<r\right\}$ has compact closure in $B$ for all $r>0$. We put $f_{n}=p_{4} X_{K_{n}+x}$. It follows immediately from Proposition 9 of [6] that $f_{n}$ is twice $H$ differentiable on $B$ and that $0 \leqq f_{n} \leqq 1,\left|D f_{n}\right| \leqq \frac{1}{2}$ on $B$.

Since each norm $\|\cdot\|_{j}$ is a continuous tame norm on $B$ and the equality

$$
p_{s}\left(K_{n}\right)=\lim _{m \rightarrow \infty} p_{s}\left\{\sum_{1}^{m}\|y\|_{j}<n\right\}
$$

holds for $s>0$, it is straightforward to show that $p_{s}\left(K_{n}\right)$ is equal to the Wiener measure (variance parameter $s$ ) of the ball of radius $n$ in the abstract Wiener space $\left(H, B^{\prime}\right)$, where $B^{\prime}$ is the completion of $H$ in the norm $\sum_{1}^{\infty}\|\cdot\|_{j}$. Hence, there holds

$$
\lim _{n \rightarrow \infty} p_{s}\left(K_{n}\right)=1
$$

for each $s$. Choose $m$ such that $p_{4}\left(K_{m}\right)>\frac{4}{5}$. Suppose $n>m$. If $k$ is in $K_{n-m}$, then

$$
f_{n}(x+k)=p_{4}\left(K_{n}-k\right) \geqq p_{4}\left(K_{m}\right)>\frac{4}{5} .
$$

Similarly if $k$ is not in $K_{n+m}$, then $f_{n}(x+k)<\frac{1}{5}$. Choose a $C^{2}$ function $\alpha: R \rightarrow[0,1]$ such that $\alpha(r)=0$ if $|r|<\frac{1}{4}$ and $\alpha(r)=1$ if $|r|>\frac{4}{5}$, and $\left|\alpha^{\prime}(r)\right| \leqq 2$ for all $r$. We put $g_{n}=\alpha f_{n}$. The function $g_{n}$ is twice $H$ differentiable by the chain rule and $\left|D g_{n}\right| \leqq 1$. Also if $y$ is in $K_{n-m}+x, g_{n}(y)=1$, and if $y$ is not in $K_{n+m}+x, g_{n}(y)=0$. Hence, $g_{n}$ has compact support, and by (2), $g_{n}$ converges to one almost everywhere with respect to $p_{t}(x, \cdot)$. Moreover, since the norm $\sum_{1}^{\infty}\|\cdot\|_{j}$ is dominated by the Hilbert norm on $H$, if $y$ is in $K_{n-m}-x$, then $y+h, h$ in $H$, is in $K_{n-m}-x$ for $|h|$ sufficiently small. But $g_{n}$ is constant on this set and so $D g_{n}(y)=0$. Thus, $\left|D g_{n}\right|$ converges to zero almost everywhere with respect to $p_{t}(x, \cdot)$.

Now suppose that $S$ is an $H-C^{1}$ surface in $B$ and that $y$ is in $S$. Let $h$ be a unit normal at $y$ and $W$ be a coordinate neighborhood of $y$ as in Definition 3. $J$ will denote the projection onto the null space $L$ of $N_{y}$. Let $\sigma_{t}(x, \cdot)$ denote the normal surface measure on $S$. To prove that $g_{n}$ converges to one almost everywhere with respect to $\sigma_{t}(x, \cdot)$ it suffices to show that this convergence holds for normal surface measure restricted to $W$. By Definition 6, this is equivalent to showing that the sequence $\left\{g_{n}\left(J^{-1}\right)\right\}$ converges to one almost everywhere with respect to Wiener measure $p_{t}^{\prime}(J x, \cdot)$ on $L$ restricted to $J(W)$.

By an argument similar to the one by which we derived (2) one may show that the sequence $\left\{K_{n} \cap L\right\}$ increases to a set which has $p_{s}^{\prime}$ measure equal to one for any $s>0$. Hence we obtain

$$
\lim _{n \rightarrow \infty} p_{s}^{\prime}\left(K_{n} \cap J(W)-J x\right)=p_{s}^{\prime}(J x, J(W)) .
$$


For a positive integer $m$ suppose that $l$ is in $K_{m}+J x \cap J(W)$. Now $J\left(J^{-1} l-x\right)$ $=l-J x$ and this vector is in $K_{m}$. Also, for any $z$ in $B, z-J z$ is in $\operatorname{span}\{h\}$. But, since $\sum_{1}^{\infty}\|h\|_{j}$ is finite, $\sum_{1}^{\infty}\left\|J^{-1} l-x\right\|$ is finite by the triangle inequality. Hence $J^{-1} l$ is in $K_{n}+x$ for $n$ sufficiently large. Now $g_{n}$ is identically one on $K_{n}+x$, and so $g_{n}\left(J^{-1} l\right)$ converges to one for any $l$ in $K_{m}+J x \cap J(W)$. By (3) then, $g_{n} J^{-1}$ converges to one almost everywhere on $J(W)$.

Definition 10. Let $(H, B)$ be an abstract Wiener space. A continuous linear operator $T: B \rightarrow B^{*}$ with finite dimensional range is said to be a test operator.

REMARK 6 . Let $T$ be a test operator for $(H, B)$. If $F: B \rightarrow B$ is any map, then the function $y \rightarrow\langle T F(y), y\rangle$ is defined on $B$. Suppose that $A: H \rightarrow H$ is a bounded operator. We shall denote by trace $[T A]$ the trace of the operator $T A$ where $\operatorname{Im} T$ is identified with a subspace in $H$. Other properties of test operators are developed in Definition 6 of [6].

Theorem 2 (Divergence Theorem). Let $V$ be a set with an $\mathrm{H}-\mathrm{C}^{1}$ boundary in an abstract Wiener space $(H, B)$. Let $F: V \cup \partial V \rightarrow H$ be a measurable function which is $H$ differentiable on $V$ and $H$ continuous at the boundary of $V$. Assume also that $D F: V \rightarrow L(H, H)$ is $H$ continuous into the weak operator topology.

If for fixed $x$ in $B$ and $t>0$ and each element $h$ in $H$ the functions $(F(y), h)^{2}$ and $|D F(y)(h)|$ are $p_{t}(x, \cdot)$ integrable on $V$ and the function $|F(y)|$ is integrable with respect to normal surface measure $\sigma_{t}(x, \cdot)$ on $\partial V$, then for any test operator $T$ on $B$ there holds

$$
\int_{V}\left\{\operatorname{trace}[T D F(y)]-t^{-1}\langle T F(y), y-x\rangle\right\} p_{t}(x, d y)=\int_{\partial V}\langle T F(y), n(y)\rangle \sigma_{t}(x, d y) .
$$

Proof. Let $V$ be an open set with $H-C^{1}$ boundary and suppose that $z$ is in $\partial V$. By Definition 3 and the proof of Proposition 2 we may choose an open ball $U$ centered at $z$ and an $H$ - $C^{1}$ function $g$ such that $V \cap U=\{y \in U: g(y)<0\}$, the set $W=\partial V \cap U$ is a coordinate neighborhood for $z$, and $\langle h, D g(y)\rangle>0$ for $y$ in $U$, where $h=n(z)$ is the outward normal for $V$ at $z$.

Let $k$ be an element of $B^{*}$ with unit $H$ norm. We show that the statement of the theorem holds for the test operator $T=\langle k, \cdot\rangle k$ and a vector field $F$ of the form $F(y)=f(y) k$, where $f$ is scalar-valued. We assume that $F$ satisfies the hypotheses in the statement of the theorem, and we assume in addition that the support of $f$ is contained in $U$.

We put $K=\operatorname{span}\{h, k\}$. Since $K$ is a subspace of $B^{*}, B=K \oplus L^{\prime}$ where $L^{\prime}$ is the annihilator of $K$ in $B$. By Remark $1, p_{t}=p_{t}^{\prime} \times \mu_{t}$ where $p_{t}^{\prime}$ is Wiener measure on $L^{\prime}$ and $\mu_{t}$ is Gauss measure on $K$. We use this product decomposition to consider the expression

$$
\int_{V}\left\{(D f(y), k)-t^{-1} f(y)\langle k, y-x\rangle\right\} p_{t}(x, d y)
$$


By Remark 1, the orthogonal projection of $H$ onto $K$ extends to a continuous projection $N^{\prime}$ of $B$ onto $K$. We put $J^{\prime}=I-N^{\prime}$. Then $J^{\prime}$ is a projection of $B$ onto $L^{\prime}$. Moreover, it is easy to see that for $x$ in $B, p_{t}(x, \cdot)=p_{t}^{\prime}\left(J^{\prime} x, \cdot\right) \times \mu_{t}^{\prime}\left(N^{\prime} x, \cdot\right)$. Put $y=l+v$ where $l$ is in $L^{\prime}$ and $v$ is in $K$. Then (4) is equal to the integral with respect to $p_{t}^{\prime}\left(J^{\prime} x, \cdot\right)$ of the expression

$$
\int_{g(l+v)<0}\left\{(D f(l+v), k)-t^{-1} f(l+k)\left\langle k, v-N^{\prime} x\right\rangle\right\} \mu_{t}\left(N^{\prime} x, d v\right)
$$

provided that the integrand in (4) is an integrable function. Now from the definition of $F,|(D f(y), k)|=|D F(y)(k)|$ and this function is integrable on $V$ by assumption. Also,

$$
\begin{aligned}
\int_{V}|f(y)\langle k, y-x\rangle| p_{t}(x, d y) & \leqq\left\{\int_{V} f^{2}(y) p_{t}(x, d y)\right\}^{1 / 2}\left\{\int_{B}\langle k, y\rangle^{2} p_{t}(d y)\right\}^{1 / 2} \\
& =t^{-1 / 2}\left\{\int_{V}(F(y), k)^{2} p_{t}(x, d y)\right\}^{1 / 2}
\end{aligned}
$$

and the last integral is finite by assumption. We also note that for any $l$, (5) exists because the integrand is a continuous function and has compact support in $K$.

For fixed $l$ we put $\alpha(v)=g(l+v)$. By the choice of $g, \alpha$ is $C^{1}$ in a neighborhood of the support of the integrand in (5). Moreover, for $v$ in this neighborhood, $l+v$ is in $U$ and so

$$
(\nabla \alpha)(v) \cdot h=(D g(l+k), h)>0 .
$$

Using the inner product structure on $K$, we have the equivalent expression

$$
\begin{aligned}
(2 \pi t)^{(-\operatorname{dim} K) / 2} \int_{\alpha(v)<0}\left\{(D f(l+v), k)-t^{-1} f(l+k)\left(k, v-N^{\prime} x\right)\right\} \\
\times \exp \left[-(2 t)^{-1}\left|v-N^{\prime} x\right|^{2}\right] d v
\end{aligned}
$$

for (5), where $d v$ is Lebesgue measure on $K$. Now the integrand is the divergence of the vector field

$$
f(l+v) \exp \left[-(2 t)^{-1}\left|v-N^{\prime} x\right|^{2}\right] k
$$

on $K$. Hence, by the classical divergence theorem, expression (6) is equal to

$$
\int_{\alpha(s)=0} f(l+s)\left(k, n^{\prime}(s)\right)(2 \pi t)^{(-\operatorname{dim} K) / 2} \exp \left[-(2 t)^{-1}\left|s-N^{\prime} x\right|^{2}\right] d s
$$

where $d s$ is (Lebesgue) surface measure on the surface $\{\alpha=0\}$. Although $\{\alpha=0\}$ does not bound an open set in $K$, the fact that $\nabla \alpha \cdot h>0$ in a convex neighborhood of the support of $f(l+v)$ allows one to apply the divergence theorem.

Now by the chain rule, $n^{\prime}(s)=\left|N^{\prime} D g(l+s)\right|^{-1} N^{\prime} D g(l+s)$. Also, $N_{z}$, the normal projection for $\partial V$ at $z$, is given by $\langle h, \cdot\rangle h$, and it follows from the implicit function theorem that the restriction of $J_{z}$ to $\{\alpha=0\}$ is a homeomorphism into $L_{z} \cap K$. Here, $L_{z}$ is the null space of $N_{z}$. Thus, we may use the local formula for $d s$ which defines 
$d s$ as a transformation of Lebesgue measure on $L_{z} \cap K$. When $d s$ is so represented, (7) becomes an integral with respect to Gauss measure on $L_{z} \cap K$. If this expression is integrated with respect to $p_{t}^{\prime}\left(J^{\prime} x, d l\right)$ on $L^{\prime}$, one obtains, using the formula in Definition 6, the expression

$$
\int_{\partial V} f(y)\langle k, n(y)\rangle \sigma_{t}(x, d y) .
$$

Thus, we have shown that the divergence theorem holds for the vector function $F(y)=f(y) k$ and the test operator $T=\langle k, \cdot\rangle k$.

We now show that the divergence theorem holds for $f(y) k$ where $f(y) k$ satisfies that hypotheses of the theorem and the support of $f$ is compact in $B$. For each $z$ in $\partial V$, let $U_{z}, g_{z}$ be chosen as in the argument above. For $z$ not in $\partial V$, let $U_{z}$ be an open ball centered at $z$ not intersecting at $\partial V$ and put $g_{z} \equiv 1$ on $U_{z}$. The family $\left\{U_{z}\right\}$ covers $B$ and by Lemma 2.1 there is an $H-C^{1}$ partition of unity $\left\{\gamma_{n}\right\}$ which is subordinate to this cover. For fixed $n$ we consider the vector field $\gamma_{n}(y) f(y) k$. Since the support of $f$ is compact, the function $D \gamma_{n}$ is bounded in Hilbert norm on this set. It follows that $F=\gamma_{n} f k$ satisfies the hypotheses for the theorem and that the support of $F$ is contained in some $U_{z}$. Hence, by the argument above, the theorem holds for $F$.

Now, using the compactness of the support of $f$ and the definition of an $\mathrm{H}-\mathrm{C}^{1}$ partition of unity, one can easily see that the divergence theorem holds for the vector field $\sum \gamma_{n} f k=f k$ and test operator $T=\langle k, \cdot\rangle k$.

Next, we consider $F(y)=f(y) k$ where no assumption concerning the support of $f$ is made. We choose a sequence of twice $H$ differentiable functions $\left\{g_{n}\right\}$ which have the properties asserted in Lemma 2.2. In particular, $g_{n}$ has compact support, $g_{n}$ and $D g_{n}$ are bounded, and by the integrability assumptions made on $F$, the divergence theorem holds for each $F_{n}(y)=g_{n}(y) f(y) k$ and $T=\langle k, \cdot\rangle k$. Moreover, upon taking limits in the integral identity, one obtains the identity for $F$.

Finally, let $F$ be a vector function satisfying the hypotheses for the theorem, and let $T$ be an arbitrary test operator. By Definition 6 of [6], there holds $T y$ $=\sum_{1}^{n}\left\langle e_{j}, y\right\rangle T e_{j}$ where $\left\{e_{j}\right\}_{1}^{n}$ is a subset of $B^{*}$ which is orthonormal in $H$. Hence, the integral

$$
\int_{\partial V}\langle T F(y), n(y)\rangle \sigma_{t}(x, d y)=\sum_{1}^{n} \int_{\partial V}\left(F(y), e_{j}\right)\left\langle n(y), T e_{j}\right\rangle \sigma_{t}(x, d y)
$$

exists by the assumptions on $F$. Also, we have shown that for each $j$, the divergence theorem holds for the vector field $F_{j}(y)=\left(F(y), e_{j}\right) T e_{j}$ and the test operator $T_{j}=\left|T e_{j}\right|^{-2}\left\langle T e_{j}, \cdot\right\rangle T e_{j}$. Upon adding the integral identities one obtains the identity for $F$ and $T$. This concludes the proof of the theorem.

Definition 11. Let $(H, B)$ be an abstract Wiener space and $U$ be an open subset of $B$. A function $F: U \rightarrow H$ has finite divergence at $x$ in $U$ if $F$ is $H$ differentiable at $x$ and $D F(x)$ is an operator on $H$ of trace class. For such a map the divergence of $F$ at $x$ is the quantity trace $[D F(x)]$, and the divergence is denoted by $(\operatorname{div} F)(x)$. 
It is easily seen that the above expression coincides with the usual definition of divergence if the space $H$ is finite dimensional.

Corollary 2.1. Let $V$ be a set with an $H-C^{1}$ boundary in the space $(H, B)$. Assume that $F: V \cup \partial V \rightarrow H$ is a measurable $H$ continuous vector field with finite divergence on $V$ and that $F$ is uniformly bounded in $B^{*}$ norm on $V$. If for $x$ in $B$ and $t>0$ the function $|F|$ is $\sigma_{t}(x, \cdot)$ integrable on $\partial V$, and the trace class norm of $D F$ is $p_{t}(x, \cdot)$ integrable on $V$, then there holds

$$
\int_{V}\left\{(\operatorname{div} F)(y)-t^{-1}\langle F(y), y-x\rangle\right\} p_{t}(x, d y)=\int_{\partial V}\langle F(y), n(y)\rangle \sigma_{t}(x, d y) .
$$

Proof. It follows from an argument in the proof of Lemma 2.2 that there is a sequence $\left\{P_{n}\right\}$ of test operators on $B$ such that the restriction of $P_{n}$ to $H$ is an orthogonal projection and $\left\{P_{n}\right\}$ converges strongly to the identity map on $H$. We apply Theorem 2 to $F$ and a test operator $P_{n}$. Now $\lim _{n \rightarrow \infty} \operatorname{trace}\left[P_{n} D F(y)\right]$ $=(\operatorname{div} F)(y)$ for each $y$, and also $\lim _{n \rightarrow \infty}\left\langle P_{n} F(y), n(y)\right\rangle=\langle F(y), n(y)\rangle$. Moreover, since $F$ is bounded in $B^{*}$ norm,

$$
\left|\langle F(y), y-x\rangle-\left\langle P_{n} F(y), y-x\right\rangle\right| \leqq M\left\|\left(I-P_{n}\right)(y-x)\right\| .
$$

By an argument in [6, p. 174], the integral of the right-hand expression relative to the measure $p_{t}(x, \cdot)$ converges to zero as $n \rightarrow \infty$. Hence, we may apply the dominated convergence theorem to the integrals in the identity obtained from Theorem 2. The resulting identity is the desired one.

Corollary 2.1 is related to a result established by Stengle [9] for Gaussian probability measures on the space $C[0,1]$. Let $C$ denote the Banach space consisting of all continuous real-valued functions on $[0,1]$ which vanish at zero. The indefinite integral defines an embedding of the space $L^{2}(0,1)$ into $C$. It is shown in [4] that the pair $\left(L^{2}(0,1), C\right)$ is an abstract Wiener space and that the measure $p_{1}$ carried by $C$ is the canonical Wiener measure. Now $p_{1}$ defines a measure on $C[0,1]$, and Corollary 4 in [9] for this measure is comparable to Corollary 2.1 above. However, the hypotheses in Corollary 4 are quite different from those in Corollary 2.1. This difference arises from the fact that surface measure is not used in [9]. In Corollary 4, a conditional expectation function is used in place of a surface integral.

6. Examples. Let $H$ be a real Hilbert space and let $A: H \rightarrow H$ be a one-to-one operator of Hilbert-Schmidt type. The norm $\|x\|=|A x|$ is a measurable norm by Theorem 2 of [4]. The space $B$ obtained by completing $H$ in this norm is itself a Hilbert space, for $\|x\|$ arises from an inner product $[x, y]=(A x, A y)$ on $H$. It is well known that the square of a Hilbert norm is infinitely Fréchet differentiable. Hence, the set $\{x \in B:\|x\|=r\}$ is a differentiable surface in $B$ for each $r>0$. By Remark 2, this set is an $H-C^{1}$ surface. 
It is an interesting fact that the above surfaces have finite normal surface measure for any parameters $x$ and $t$. This is a consequence of the following more general result which we derive with the use of the divergence theorem.

Let $(H, B)$ be an abstract Wiener space and $K$ be a two-dimensional subspace of $B^{*}$. By Remark 1 there are projections $Q$ and $J$ of $B$ onto $K$ and $\operatorname{ker} Q$ respectively, and $L=\operatorname{ker} Q$ carries a family $\left\{p_{t}^{\prime}\right\}$ of Wiener measures. Now the corollary to Proposition 1 is applicable to the space $\left(K^{\perp}, L\right)$. That is, for a given $\varepsilon>0$, there is a function $f: L \rightarrow R$ satisfying a Lipschitz condition such that the function $p_{t}^{\prime} f$ has a nonzero $K^{\perp}$ derivative on $\left\{l \in L: \varepsilon<\|l\|<\varepsilon^{-1}\right\}$ for $t$ small, and $p_{t}^{\prime} f$ approximates the norm on this set. Hence, if $r>0$ is given, for a suitable $t>0$ and $f$, the function $g(x)=|Q x|^{2}+p_{t}^{\prime} f$ has a nonzero $H$ derivative on the nonempty set

$$
\{x \in B: r / 2 \leqq g(x) \leqq r\} .
$$

Moreover, by the choice of $f$, the set $V=\{x \in B: g(x)<r\}$ has an $H$ - $C^{1}$ boundary. In addition, the vector field $F(x)=D g(x)$ has finite divergence on $V \cup \partial V$ by [6, Theorem 3] and the trace class norm of $F$ is a uniformly bounded continuous function of $x$. By composing $g$ with a suitable $C^{1}$ function $\alpha: R \rightarrow R$, one obtains a vector field $F^{\prime}(x)=\alpha(g(x))|D g(x)|^{-2} F(x)$ which has finite divergence on $V$ and is such that $F^{\prime}(x)=|D g(x)|^{-2} D g(x)$ if $g(x)=r$.

Let $\left\{g_{n}\right\}$ be a sequence of twice $H$ differentiable functions with the properties asserted in Lemma 2.2. Since $g_{n}$ has compact support, each vector field $g_{n} F^{\prime}$ satisfies the hypotheses for Corollary 2.1. Hence, we obtain the identity

$$
\int_{V}\left\{\left(\operatorname{div} g_{n} F^{\prime}\right)(y)-t^{-1} g_{n}\left\langle F^{\prime}(y), y-x\right\rangle\right\} p_{t}(x, d y)=\int_{\partial V} g_{n}(y)|D g(y)|^{-1} \sigma_{t}(x, d y)
$$

for any $x$ and $t$. Now by the definition of $F^{\prime}$ there holds

$$
\left|\left(\operatorname{div} g_{n} F^{\prime}\right)(y)\right| \leqq c|D g(y)|^{-2} \text { and }\left|\left\langle F^{\prime}(y), y-x\right\rangle\right| \leqq c|D g(y)|^{-2}
$$

for some $c>0$. But,

$$
\begin{aligned}
\int|D g(y)|^{-2} p_{t}(x, d y) & \leqq \int|Q y|^{-2} p_{t}(d y) \\
& =(2 \pi t)^{-1} \int_{R^{2}}|v|^{-2} \exp \left[-(2 t)^{-1}|v|^{2}\right] d v<\infty .
\end{aligned}
$$

Hence, the left-hand expression in (8) remains bounded as $n \rightarrow \infty$. It follows that the integral $\int_{\partial V}|D g(y)|^{-1} \sigma_{t}(x, d y)$ is finite. Since $|D g(y)|$ is bounded, this implies that $\sigma_{t}(x, \partial V)$ is finite. Thus, we have shown that in any abstract Wiener space, there exist nonempty bounded sets whose boundaries carry finite surface measure.

In an example above we considered a norm given by $\|x\|=|A x|$ where $A$ is a Hilbert-Schmidt operator on a Hilbert space $H$. The argument above may be applied to the function $g(x)=\|x\|^{2}$. One has only to note that an eigenvector of the operator $A^{*} A$ on $H$ is in $B^{*}$. Then if $K$ is the span of any two eigenvectors, the 
identity $g(x)=\|Q x\|^{2}+\|J x\|^{2}$ holds for the projections $Q$ and $J$ onto $K$ and ker $Q$. One may verify directly that the function $|D g(y)|^{-1}$ is unbounded on the sphere $\{x \in B:\|x\|=r\}$. However, by the argument above it is integrable relative to any normal surface measure.

\section{REFERENCES}

1. R. Bonic and J. Frampton, Differentiable functions on certain Banach spaces, Bull. Amer. Math. Soc. 71 (1965), 393-395. MR 30 \#2310.

2. J. Dieudonné, Foundations of modern analysis, Pure and Appl. Math., vol. 10, Academic Press, New York, 1960. MR 22 \#11074.

3. I. M. Gel'fand and N. Ja. Vilenkin, Generalized functions. Vol. 4: Some applications of harmonic analysis, Fizmatgiz, Moscow, 1961; English transl., Academic Press, New York, 1964. MR 26 \#4173; MR 30 \#4152.

4. L. Gross, Measurable functions on Hilbert space, Trans. Amer. Math. Soc. 105 (1962), 372-390. MR 26 \#5121.

5. - Abstract Wiener spaces, Proc. Fifth Berkeley Sympos. Math. Statist. and Probability (Berkeley, Calif., 1965/66), vol. II: Contributions to Probability Theory, part 1, Univ. California Press, Berkeley, Calif., 1967, pp. 31-42. MR 35 \#3027.

6. - Potential theory on Hilbert space, J. Functional Analysis, 1 (1967), 123-181. MR 37 \#3331.

7. J. Kurzweil, On approximation in real Banach spaces, Studia Math. 14 (1954), 214-231. MR 16, 932.

8. S. Lang, Introduction to differentiable manifolds, Interscience, New York, 1962; Russian transl., Moscow, 1967. MR 27 \#192.

9. G. Stengle, A divergence theorem for Gaussian stochastic process expectations, J. Math. Anal. Appl. 21 (1968), 537-546. MR 37 \#970.

10. J. H. M. Whitfield, Differentiable functions with bounded nonempty support on Banach spaces, Bull. Amer. Math. Soc. 72 (1966), 145-146. MR 32 \#1547.

Department of Mathematics, University of New Mexico, Albuquerque, New Mexico 87106 